\title{
OFERTA Y DEMANDA EN EL SECTOR SANITARIO: UN ANALISIS DESDE LA ECONOMIA DE LA SALUD
}

\author{
Karina Temporelli*
}

\begin{abstract}
Resumen
El análisis de la oferta y la demanda de servicios sanitarios es el paso previo para la correcta implementación de políticas sanitarias que respondan a las necesidades de la población objetivo. En el contexto del sector sanitario, estos componentes no se comportan de la misma forma que en el resto de la economía. La complejidad de la oferta sanitaria, en la que conviven instituciones públicas, privadas con objetivos de maximización de beneficios e instituciones sin fines de lucro, torna difícil definir un precio monetario único que permita una asignación correcta de recursos. Por otro lado, los problemas que se presentan en la medición de la producción dificulta la evaluación económica. Por el lado de la demanda los problemas de información, la incertidumbre y la presencia de terceros pagadores impiden que los consumidores puedan evaluar alternativas racionalmente, poniendo en muchos casos en manos de los oferentes sus decisiones de consumo. El objetivo de este trabajo es analizar las dificultades que plantean para el análisis económico las nociones de demanda y oferta de servicios sanitarios mostrando críticamente como la economía de la salud aborda esta problemática.
\end{abstract}

Clasificación JEL: I2, H4.

Palabras clave: salud - oferta de asistencia sanitaria - demanda asistencia sanitaria - fallas de mercado

\begin{abstract}
The analysis of the supply and the demand of sanitary services is the previous step for the correct implementation of sanitary policies that answer to the needs of the objective population. In the context of the sanitary sector, these components do not comport of the same form that in the rest of the economy. The complexity of the sanitary supply, where there are publics, privates and non profits institutions with diferent incentives, do difficult to define a monetary price. On the other hand the problems in the production measurement do difficult the economic evaluation. By the side of the demand the problems of information, the uncertainty, the payments realized by third that the consumers can not evaluate alternatives in rational way, putting several times his consumption decisions in hands of the suppliers. The aim of this work is to make a review to the difficulties in the analyse of demand and offer of sanitary services, showing like the health's economic analyses this problematic.
\end{abstract}

JEL Classification: I2, $\mathrm{H} 4$

Keywords: sanitary market - supply health - demand health- market failures

\footnotetext{
* Departamento de Economía, Universidad Nacional del Sur, CONICET, e.mail: ktempo@criba.edu.ar
} 


\section{INTRODUCCION}

La oferta y demanda de cualquier bien o servicio constituyen las condiciones básicas que determinan la estructura del mercado, la conducta de los agentes que intervienen y los resultados a los que se llega. Muchos economistas han centrado su atención en el análisis de estos componentes en el sector sanitario siendo uno de los temas que da origen al surgimiento de la Economía de la Salud como disciplina. Las características distintivas de este sector hacen necesario utilizar un conjunto de herramientas específicas para realizar análisis económico, sin perder de vista que nos encontramos ante un bien con ciertas particularidades que exige un análisis diferencial y multidisciplinario, que no deje de lado las cuestiones clínicas, éticas y sociales.

Se propone en este trabajo el análisis de la oferta y demanda de asistencia sanitaria utilizando una visión dinámica, en la que los sistemas de salud se constituyen a través de un conjunto de relaciones estructuradas entre dos componentes fundamentales: la demanda, constituida por la población con características especiales que enfrentan necesidades sanitarias y por lo tanto demandan servicios sanitarios y la oferta compuesta por instituciones públicas y privadas como la respuesta social organizada para satisfacer dicha demanda (Londoño, 1997).

En el primer apartado se analizan las cuestiones teóricas acerca de la demanda de asistencia sanitaria centrándose en los diferentes enfoques para su tratamiento, partiendo del modelo neoclásico tradicional, incorporando luego la noción de necesidad y por último los modelos en dos etapas.

En el apartado siguiente se analiza la oferta mostrando sus características más relevantes. Se hace hincapié en las dificultades de medición de la producción y en la incorporación tecnológica. La variación de la práctica médica presenta una dificultad adicional para el análisis económico.

Dadas las características propias de la oferta y la demanda, surge un tema extremadamente interesante para el análisis económico: la posibilidad de inducción de demanda por parte de la oferta y las consecuencias para el sector.

En el último punto se introduce brevemente el rol del estado para luego presentar las consideraciones finales.

\section{DEMANDA DE ASISTENCIA SANITARIA}

\section{I.1 El modelo neoclásico tradicional}

En el mercado de servicios sanitarios la demanda es difícil de predecir individualmente (incertidumbre) y esta sujeta a problemas de información (información asimétrica). La demanda de atención sanitaria puede derivarse de la propia función de 
producción de salud ${ }^{1}$ y genera a su vez una gran cantidad de demandas derivadas entre las cuales se identifican la de medicamentos, diagnósticos por imágenes, internación, etc. En estos casos no es el individuo el que demanda sino que lo hace a través del médico quien indica qué y cómo demandar.

En este contexto los problemas de información son importantes. El papel decisorio del paciente en este mercado no se corresponde con el papel que tiene como consumidor en el mercado de otros bienes y servicios.

Para los bienes convencionales típicos la demanda expresa la disponibilidad a pagar de los individuos. Esta disponibilidad depende de una serie de variables como el precio del bien, los precios de los demás bienes o servicios considerados, la renta del individuo o familia y toda otra serie de factores entre los que se incluyen los gustos. Cuanto más amplio es el conocimiento de los efectos de estos determinantes, mejor se explican las variaciones de consumo entre diferentes grupos o áreas de población y por lo tanto se puede predecir e influir sobre consumos futuros mediante cambios en las variables explicativas.

Desde el punto de vista teórico existen dos enfoques diferentes para el análisis de la demanda de asistencia sanitaria. El primero es el neoclásico tradicional que parte de un consumidor representativo racional que obtiene su función de demanda maximizando una función de utilidad sujeta a una restricción presupuestaria. Esta función presenta una relación inversa con respecto al precio y directa con respecto al ingreso. Dadas las características particulares del mercado es conveniente analizar los determinantes de la demanda con más detalle ya que su peso relativo puede ser diferente al de otros sectores.

El precio en este mercado es sólo una más de las variables que intervienen en la función de demanda. Es afectado por la existencia de seguros médicos, servicios gratuitos o subsidiados, con el fin de superar problemas generados por las restricciones presupuestarias individuales. En muchos casos, especialmente en la asistencia médica en hospitales públicos, el precio es cercano a cero por lo cual operan otras formas de racionamiento. La existencia de terceros pagadores (Estado, aseguradoras, seguridad social) diferencia el precio de mercado del pagado efectivamente por los demandantes, afectando las cantidades demandadas de los bienes o servicios relacionados.

Mientras que en gran parte de los análisis de demanda de bienes y servicios los cambios en los gustos se consideran constantes, en el caso de la atención médica esta simplificación no siempre es válida por la existencia constante de cambios en la tecnología, nuevos descubrimientos y terapias alternativas. La variabilidad de la práctica médica puede afectar las preferencias por tratamientos alternativos a partir de cuestiones subjetivas difíciles de explicar desde la racionalidad económica. Se debe tener presente que el proceso salud-enfermedad está influenciado por factores culturales que le dan una connotación especial al fenómeno, cuya solución depende en parte de la percepción del problema, tanto por parte del demandante como del oferente.

\footnotetext{
${ }^{1}$ Cada individuo posee una función de producción de salud cuyos determinantes son la biología, los estilos de vida, los hábitos, el medio ambiente y la asistencia sanitaria. Los individuos producen salud y demandan asistencia sanitaria cuando su stock de salud disminuye (curación) o para evitar que esto suceda (prevención).
} 
En cuanto a los precios relativos surge nuevamente la dificultad en el análisis basado en precios debido a que este no es aquí un buen racionador. En muchos casos las diferencias en precios no adquieren importancia para la elección entre alternativas. En cuanto a la tecnología de última generación, a veces sólo disponible en el sector privado y aún no incluida en la nómina de cobertura de los seguros, el precio puede desempeñar el rol que juega en otros mercados. En el resto, la elección entre tratamientos alternativos generalmente está en manos del médico, mejor informado, que en última instancia es el que sugiere la elección entre uno y otro tratamiento con algún grado de libertad del paciente. En la etapa de diagnóstico la posibilidad de elección por parte del paciente es aún menor. En algunos sectores poblacionales esto está cambiando debido a la posibilidad de acceso a información vía internet.

El ingreso tiene efectos positivos sobre la demanda de salud por varios motivos. Los individuos con ingresos más altos realizan mayor cantidad de acciones para promover la salud efectivizando más cantidad de actos tendientes a la prevención, curación y rehabilitación. En materia de promoción se observa que a mayor ingreso mayor demanda de acciones para mantenerse sano. En materia de prevención, curación y rehabilitación, los individuos con mayores ingresos tienen mayor capacidad económica e información para afrontar riesgos y responder eficazmente ante emergencias o secuelas de enfermedad. Este sector de la población es el que valora más la importancia de un tratamiento oportuno. El ingreso constituye una limitación que se intenta superar a través del aseguramiento o asistencia pública gratuita. En cuánto a la elasticidad ingreso, la literatura aún no ha encontrado acuerdo acerca de si estamos frente a un bien normal (elasticidad entre 0 y 1 ) o un bien de lujo (elasticidad mayor que 1) (Temporelli, González, 2008). La importancia de este tema radica en la magnitud del aumento previsto de los gastos sanitarios a medida que se incrementa el nivel de ingreso.

Los costos indirectos que comprenden los costos por tiempo de traslado, de tratamiento y de espera, son la señal que puede actuar como racionadora cuando el precio no es un buen indicador.

Uno de los determinantes importantes de la demanda a nivel agregado lo constituyen los consumidores potenciales, relacionado con el tamaño de la población, edad y sexo, particularmente en el caso de la atención médica donde las mujeres en edad fértil, los niños y los ancianos constituyen gran parte de la demanda de servicios médicos. También cabe resaltar que en el caso de la atención médica, las enfermedades se presentan diferencialmente según grupos etareos y condiciones socioeconómicas. El análisis de las variables demográficas es el primer paso para estimar demanda dentro del sector, sabiendo que en general estos indicadores están disponibles en mayor o menor medida para prácticamente todas las poblaciones objetivo.

Parte de los economistas de la salud plantean la posibilidad de que el análisis neoclásico tradicional hasta aquí presentado, no explique correctamente la demanda de servicios sanitarios debido a que se está ante un bien con características diferentes. A partir de estas críticas surgen una serie de nuevas percepciones acerca de la demanda de salud. 
Las características particulares de la asistencia sanitaria parten del hecho de que en general los servicios sanitarios no se desean por sí mismos, debido a que su consumo suele ser normalmente desagradable o molesto. Por otro lado la demanda de atención médica es de naturaleza exógena. Esto significa que muchas de las decisiones referidas al tipo y la cantidad de consumo sanitario son tomadas por el médico y no por el paciente. Es decir que el médico actúa como agente de la demanda del paciente, por lo que oferente y demandante coinciden, violándose una de las reglas básicas exigidas para el funcionamiento de un mercado en competencia perfecta (supuesto de independencia de oferta y demanda). La aleatoriedad de las necesidades y la ausencia de sustitutos cercanos complican aún más este análisis.

A raíz de esto surge una aproximación alternativa basada en la noción de necesidad cuyos principios generales son que el consumo de cuidados médicos responde a una necesidad y no a un deseo de los individuos, rechazando el modelo convencional del mercado como instrumento más adecuado para la asignación y distribución eficiente y equitativa de los servicios sanitarios entre la población. Es decir que cada individuo recibirá -al menos en teoría- tantos cuidados médicos como los expertos estimen que necesita y no tantos como esté dispuesto o pueda pagar (Grossman, 1972). Este modelo supone que el individuo hereda un stock de capital de salud inicial dado, que se deprecia con el tiempo y que puede ser aumentado con la inversión; esto es, el individuo o la familia puede producir salud a partir de bienes, tales como la dieta, el ejercicio, la vivienda, la atención sanitaria, etc. Por lo tanto, la demanda de servicios médico-sanitarios, como insumo para la producción de la mercancía salud, es una demanda derivada de la demanda de salud.

En este sentido los individuos demandan salud por dos razones:

-como elemento de consumo, ya que ésta entra directamente en la función de utilidad -o más correctamente, su carencia es fuente de desutilidad-.

-como elemento de inversión, puesto que el stock de salud determina la cantidad de tiempo disponible para todas las demás actividades, de mercado y no mercado.

En el modelo, el individuo adulto maximiza una función de utilidad intertemporal cuyos argumentos son los bienes de consumo y el tiempo de enfermedad a lo largo de su vida, sujeta a la inversión bruta en salud que quiera realizar y a la variación de sus activos financieros.

Del mismo surge que la demanda estructural de salud (H) (Grossman, 1972, Wagstaff, 1986) es:

$$
\mathrm{H}=\mathrm{g}(\mathrm{Pm}, \mathrm{P} f, \mathrm{Pt}, \mathrm{i}, \mathrm{t}, \mathrm{A}, \mathrm{E})
$$

donde

$P m$ es el precio de los cuidados médicos

$P f$ es el precio de los alimentos

Pt es el costo del tiempo

$i$ es el precio sombra de los activos iniciales

$t$ es la edad del individuo

$A$ son factores ambientales 
$E$ es la productividad de la inversión en salud

Dado que el individuo demanda bienes y servicios que combinados con su propio tiempo genera el estado de salud, la demanda de bienes y servicios es una demanda derivada.

La demanda derivada de cuidados médicos $(\mathrm{M})$ es:

$$
\mathrm{M}=\mathrm{m}(\mathrm{H}, \mathrm{Pm}, \mathrm{Pf}, \mathrm{Pt}, \mathrm{t}, \mathrm{A}, \mathrm{E})
$$

$\mathrm{H}$ es el stock de salud.

A partir de la demanda estructural y la demanda derivada se obtiene la forma reducida para la demanda de cuidados médicos:

$$
\mathrm{M}=\mathrm{mr}(\mathrm{Pm}, \mathrm{Pf}, \mathrm{Pt}, \mathrm{ì,} \mathrm{t}, \mathrm{A}, \mathrm{E})
$$

Por lo tanto, del modelo surge la diferencia entre la demanda por salud y la demanda por cuidados médicos.

Los resultados teóricos del modelo son los siguientes:

-la salud es un bien de capital ya que las inversiones en salud ofrecen rendimientos para más de un periodo El carácter productivo de la salud surge porque con un buen estado de salud, los individuos pueden desempeñarse de manera más efectiva en sus puestos de trabajo y en la adquisición del conocimiento.

-la salud puede ser vista como un bien de inversión dejando de lado los aspectos relacionados con el consumo para así compararla con otras formas de capital humano.

-existe una relación directa entre el "stock" de capital salud y el número de días saludables que pueda obtener el individuo; esta relación es positiva pero decreciente y el número de días saludables tiene un límite dado por el número de días en el período. Esta propuesta puede apoyar uno de los supuestos claves en el modelo de Barro (1996), donde los aportes de la salud para disminuir la depreciación del capital humano son decrecientes.

-el capital salud difiere de otras formas de capital humano. En particular, el nivel de conocimiento de la personas afecta su productividad tanto para producir bienes comerciales como bienes domésticos, mientras que su stock de capital salud determina, además de la productividad, la cantidad total de tiempo que el individuo puede gastar para obtener sus ingresos y generar bienes domésticos. Así, la salud además de afectar la tasa de salarios, principalmente afecta el tiempo perdido por estar enfermo.

-la demanda por servicios médicos es una demanda derivada de la necesidad de mejorar el stock de capital salud.

Desde el punto de vista empírico haciendo una reseña de las principales predicciones sobre el papel de las variables explicativas en la determinación de la demanda que realizó Grossman (1972), es necesario advertir que gran parte de los mismos fueron realizados con datos para Estados Unidos por lo que su aplicación inmediata sin una correcta evaluación de los sistemas locales, puede no ser correcta.

Las predicciones del enfoque de Grossman pueden resumirse de la siguiente manera: 
- En las pocas veces en que la variable "estado de salud" pudo ser incluida en las estimaciones se revela como el factor de mayor poder explicativo. Normalmente esta variable no es fácilmente observable por lo que se toman variables proxy, generalmente la edad del individuo. Al respecto está comprobado estadísticamente que la utilización sanitaria sigue una función polinómica de segundo grado, con máximo en los dos extremos y mínimo en la edad joven adulta, siendo mayor la utilización por parte de las mujeres debido a la maternidad.

- La elasticidad de los servicios médico- sanitarios con respecto al precio monetario es muy baja, incluso inferior a la unidad. Sin embargo debido a la difusión del seguro de salud -público o privado- el tiempo, como expresión del precio no monetario, ha venido a sustituir al precio monetario como elemento esencial en los modelos de demanda sanitaria.

- La elasticidad ingreso en los estudios de corte transversal tampoco es muy alta, debido asimismo a la difusión de los seguros, e incluso en algún caso se hallan elasticidades negativas para los servicios públicos y positivas para los privados.

- La demanda de servicios médico-sanitarios es bastante elástica con respecto a la cobertura de seguro. No obstante, la naturaleza de la relación entre ambas variables está en tela de juicio, algunos autores piensan que son los individuos con mayor demanda esperada de servicios médicos los que contratarán mayores niveles de cobertura.

- No hay una relación clara y única entre el nivel educativo y la utilización de servicios médico-sanitarios, aunque tiende a predominar el efecto predicho por Grossman en cuanto a que los individuos más educados demandan más salud pero menos atención sanitaria.

Hasta aquí se tienen dos alternativas de análisis de la demanda de asistencia sanitaria y cada una tiene implicancias distintas en cuanto a medidas de política económica y por lo tanto en la morfología de los mercados de asistencia sanitaria. La primera sugiere que para aumentar la demanda de salud es necesario disminuir su precio (para este enfoque demanda de salud es demanda de servicios médico-sanitarios). En la segunda, que toma al costo del tiempo como una variable explicativa importante, la baja del precio de servicios médico-sanitarios puede llevar a una disminución de la cantidad demandada dada una necesidad de mayor intensidad en términos de tiempo para obtener una unidad adicional de salud. Esto implica que la forma más efectiva y segura de aumentar la demanda de salud sería a través de la disminución de los precios que dependen del tiempo.

\section{I.3. Demanda en dos etapas}

Una alternativa de análisis más reciente define la demanda de servicios sanitarios en dos etapas (Pohlmeier and Ulrich, 1995). Esta distinción es especialmente interesante a la hora de su medición. En la primera etapa el paciente toma la decisión de realizar la primera consulta para maximizar su propia utilidad; en la segunda etapa hay una combinación entre las preferencias del paciente y del médico, quien en definitiva decide el tipo y la intensidad del tratamiento (Temporelli, 2007).

En la primera etapa el individuo ingresa en el sistema de atención médica, maximizando su propia utilidad. De esta maximización surge su primera demanda por servicios sanitarios, realizando o no una consulta al médico. Debido a la asimetría de información, delega las demandas posteriores en un agente, el médico del cuál se 
convierte en su principal. En la segunda etapa es el médico el que define las medidas a seguir convirtiéndose en el demandante de servicios de diagnóstico, medicamentos, internación y tratamientos en general. La utilidad a maximizar ahora es la del médico, que incluye como uno más de sus argumentos la utilidad del paciente. Este tipo de modelos intentan captar los problemas de asimetría de información y reflejarlos en la conducta de los agentes intervinientes.

En este sentido unas de las variables más relevantes en estos estudios es la forma de pago por los actos médicos, dado que diferentes modalidades contractuales de retribución pueden generar distintos incentivos y afectar significativamente los resultados a los que se arriba. Este tema se retomará en el apartado donde se trata la posibilidad de inducción de la demanda bajo determinadas formas de pago.

\section{OFERTA DE BIENES Y SERVICIOS SANITARIOS}

Se define a la oferta de servicios sanitarios como la disponibilidad de recursos (humanos, materiales y económicos) con que cuenta una comunidad para atender la demanda de una determinada población. La oferta refleja los esfuerzos que se realizan para enfrentar los problemas de salud de una población y está vinculada en su dimensión y características con el grado de desarrollo alcanzado.

Incluye el análisis de la producción de servicios de salud por tipo de programa de salud, institución de salud, funciones de producción e insumos requeridos para satisfacer la demanda de salud, como así también el análisis económico de la respuesta social organizada para proveer de servicios de salud (sistemas de salud).

Asimismo, estudia la dinámica de factores estructurales dentro de la prestación de servicios, tales como la productividad, el patrón de equipamiento y los esquemas de organización y financiamiento. Incluye también el análisis de las economías de escala y los aspectos relacionados con la tecnología.

Se puede definir la producción de bienes y servicios como el proceso a partir del cual insumos tales como recursos materiales, trabajo humano, tecnología médica, infraestructura física, capital, etc., son utilizados para generar productos, bienes y servicios dirigidos a la salud.

La teoría económica enfrenta aquí también una serie de problemas como resultado de las características intrínsecas del sector que dificulta la evaluación económica:

-en la atención médica no hay acuerdos en forma de medir el producto final de los servicios de salud. Tradicionalmente se han tomado como unidades de medida las consultas otorgadas, los egresos, las intervenciones quirúrgicas, etc. pero no existe consenso al respecto. Especialmente en el sector hospitalario, es complicado medir el producto, y en caso de hacerlo se dificulta la comparación de los resultados ya que las instituciones difieren en el tipo de casos que tratan.

-el producto es medido en cantidad y no siempre se toma en cuenta la calidad. Aquí las normas cuantitativas y cualitativas de la atención médica están formuladas en términos de servicios. Por ejemplo un médico puede dar una atención apropiada técnicamente, 
pero con una relación médico paciente poco satisfactoria para alguna de las partes o puede dar una cantidad de consultas diarias que sobrepase el estándar indicado, pero con una técnica deficiente.

-es difícil tratar las diferencias en calidad. Deficientes medidas de calidad pueden arrojar resultados incorrectos en cuánto a economías o deseconomías de escala.

-no hay parámetros de productividad estandarizados. Las evaluaciones de los servicios se basan mayormente en las historias clínicas siendo factible que éstas no contengan los datos suficientes para realizar las valoraciones correspondientes.

-no hay consenso en las unidades de medida de insumos, productos (bienes y servicios), y estándares de productividad para las unidades de atención médica, por lo que las variaciones en la cantidad de atención recibidas por presupuesto gastado no se pueden comparar.

Además de los temas hasta aquí tratados, dentro del análisis de la oferta dos temas pueden considerarse relevantes: la variación de la práctica médica y la incorporación tecnológica.

Parte de los problemas que dificultan la evaluación económica se presentan por la existencia de una gran variabilidad en la práctica médica, inconveniente importante a la hora de medir y comparar resultados.

Si bien es esperable la existencia de alguna variabilidad en los actos médicos entre regiones, la realidad nos muestra que esta variabilidad es bastante grande. El análisis de esto "...refiere al interrogante de por que los médicos hacen lo que hacen y sobre cual es la naturaleza del saber que guía sus acciones" (Tafani, 2000). Esto es especialmente interesante para la economía ya que plantea el problema de la productividad de los servicios y dificulta la obtención de una medida de dicha productividad.

Entre las causas que normalmente se atribuyen a la existencia de este fenómeno podemos citar:

- incertidumbre médica en el diagnóstico y tratamiento que se traduce en diferentes estilos de práctica, reflejando su propia estructura de creencias sobre los beneficios de tratamientos alternativos. Los estudios empíricos al respecto tratan de captar diferenciales en la educación de los médicos y otras cuestiones particulares.

- diferencia en la cobertura de seguros en distintas áreas geográficas.

- la cantidad, grado de entrenamiento y la composición generalista -especialista del cuerpo médico.

A pesar de existir una amplia literatura sobre este tema, no es posible determinar a qué se debe esta gran variabilidad y cuál es la cantidad y tipo apropiado de tratamiento en cada situación. Tampoco existe acuerdo entre los profesionales de cuál es la tasa adecuada de intervención para muchos procedimientos.

Podemos decir que mucho de lo que constituye la medicina moderna no ha sido probada en forma científica y que su productividad marginal o eficacia en restaurar o aumentar la salud permanece como una pregunta abierta (Tafani, 2000). 
Esto afecta directamente al comportamiento de los mercados de asistencia sanitaria al introducir cierta incertidumbre en el grado de contribución de la asistencia médica sobre la salud, dificultando la medición de la eficiencia.

Dentro del análisis de la oferta de productos y servicios sanitarios el cambio tecnológico, que puede ser visto como un input más dentro de la función de producción, juega un rol importante, considerado por muchos autores en gran parte el causante del aumento de los gastos sanitarios.

La teoría también sugiere que el cambio tecnológico puede ser afectado por la cobertura de seguro. Con una mayor cobertura mejora la rentabilidad de las innovaciones que incrementan costos en contra de las que los reducen. Esta relación es bidimensional ya que la tecnología provoca un incremento en la demanda de seguros (Weisbrod, 1991).

Aunque la causalidad entre incorporación tecnológica y costos sea cierta, no se entiende con exactitud el mecanismo de funcionamiento de la misma. No es posible afirmar que todo avance tecnológico necesariamente incremente el gasto en salud, pero tampoco que lo disminuya. Una tecnología que aumente el costo de atención de una enfermedad particular tiende a incrementar la demanda de seguros de salud, mientras que una que los disminuya tiende a bajar la demanda de seguros. Esto último sugiere la siguiente hipótesis: el crecimiento en la cobertura de los seguros indica que el avance tecnológico de las últimas décadas ha incrementado el gasto en el cuidado de la salud asociado a varias enfermedades.

Dada la importancia de la tecnología y la capacidad en muchas situaciones de modificar la configuración de la oferta, y por lo tanto afectar la demanda, la correcta evaluación de las mismas es una tarea fundamental.

\section{II.1 La demanda inducida por la oferta}

No podemos hablar de demanda y oferta sanitaria sin analizar, al menos brevemente uno de los temas que ha convocado a los economistas de la salud desde hace décadas: la oferta inductora de demanda. En una primera aproximación, se habla de inducción de demanda siempre que la cantidad demandada real en el mercado difiera de la que los consumidores adquirirían si tuviesen plena información.

Esta particularidad del sector sanitario se basa en una situación de oportunismo por parte de los productores, los cuales poseen un incentivo para variar la demanda de los consumidores con la finalidad de maximizar su utilidad. Esta posibilidad está dada por la falta de información de los pacientes, y se verá potenciada si, una tercera parte (entidad aseguradora o estado) ajena al propio paciente y al proveedor, cubre el costo total. En este caso, los incentivos de proveedores y consumidores son coincidentes: cada uno de ellos tiene posibilidad de actuar bajo su posición preferente frente al principal de la relación, que es la parte con menos información tanto respecto al paciente como al médico. 
En los mercados tradicionales, los consumidores, con distinto grado de información, deciden qué bienes y servicios consumir. En el mercado de servicios médicos, el paciente no elige directamente qué estudio de diagnóstico, que tratamiento, o qué medicamento consumir, sino que la demanda la efectiviza el médico.

Este fenómeno es analizado en primer lugar por Shain y Roemer (1959) y Roemer (1961). En estos trabajos empíricos se encuentra una alta correlación entre la disponibilidad de camas en los hospitales generales, medida en camas hospitalarias por cada mil habitantes, y las tasas de utilización, medidas en días de estadía cada mil habitantes. Comienza a denominarse a esta situación ley de Roemer o efecto Roemer.

Desde allí una larga lista de autores comienza a ocuparse del tema analizando por un lado la posibilidad de que exista o no inducción y bajo que condiciones, mientras que otro grupo se ocupa de analizar cuál es límite de la misma (Temporelli, 2000).

Se incorporan variables como la ética profesional, la cantidad de médicos por habitante y los procesos de vigilancia entre otros pero en general se coincide en que en mayor o menor medida, el mecanismo de retribución por servicios sanitarios determina una parte importante de los incentivos de la oferta para inducir demanda.

Cada forma de pago genera un conjunto de incentivos diferentes. En el caso del pago por prestación existe una clara predisposición a inducir, aunque presenta como ventaja que en general la satisfacción del paciente es mayor hasta que los costos de oportunidad se convierten en relevantes. En cuanto a la cápita y el salario en principio parecen no presentar incentivos a sobredemandar consultas médicas, aunque no está claro lo que sucede con otras demandas derivadas En general el problema de estos sistemas es que tiende a disminuir la percepción de calidad de los pacientes (Temporelli, 2001).

De una u otra forma es claro que la oferta posee información que la demanda en principio desconoce, poniéndose en una posición de superioridad que hace difícil la negociación en igualdad de condiciones. Esta situación se agrava por las dificultades en la medición objetiva de resultados y de la eficiencia técnica de los tratamientos. Esto, unido a que en sistemas sanitarios basados en el aseguramiento, no solo el que demanda (médico) no es el que consume (paciente) sino que existe un tercer agente que se ocupa de realizar el pago (aseguradora). Es por eso que en este mercado el Estado, más allá de la provisión de bienes públicos y preferentes, se convierte en un jugador importante, independientemente de cuán público o privado sea el sistema sanitario en cuestión.

\section{EL ROL DEL ESTADO EN EL SECTOR}

Otro de los temas neurálgicos para la economía de la salud ha sido la composición pública-privada y el rol del Estado en el sector.

Se ha demostrado desde el punto de vista teórico, como así también en las experiencias prácticas más liberales, que el Estado debe participar activamente en el sector sanitario. En primer lugar debe ofrecer alternativas cuando el mercado no está lo suficientemente desarrollado para darlas, tratando de disminuir los efectos nocivos de 
las fallas del mercado. En segundo lugar asegurando la igualdad de oportunidades a través de la redistribución de los recursos.

Existen distintas formas de intervención estatal. El objetivo es replantear las formas y los medios más adecuados a fin de lograr la máxima equidad y eficiencia dadas las particularidades de cada sociedad (condiciones básicas de oferta y demanda). Existen dos alternativas aunque puede variar su implementación (ver Cuadro 1): financiar la oferta y subsidiar la demanda.

Cuadro 1- Características de cada modelo

\begin{tabular}{|c|c|c|c|c|}
\hline Modelos & ¿Quién paga? & $\begin{array}{c}\text { Progresividad del } \\
\text { pago }\end{array}$ & Destino del gasto & $\begin{array}{c}\text { Prestación de los } \\
\text { servicios }\end{array}$ \\
\hline $\begin{array}{c}\text { Subsidio a la } \\
\text { demanda }\end{array}$ & $\begin{array}{c}\text { Cuentas } \\
\text { personales }\end{array}$ & $\mathrm{Si}$ & $\begin{array}{c}\text { Finalidades } \\
\text { sociales con libre } \\
\text { elección } \\
\text { (subsidio a la } \\
\text { demanda) }\end{array}$ & $\begin{array}{c}\text { Mercado } \\
\text { regulado Estado } \\
\text { "testigo" }\end{array}$ \\
\hline Estado benefactor & Impuestos & $\mathrm{Si}$ & $\begin{array}{c}\text { Finalidades } \\
\text { sociales sin libre } \\
\text { elección } \\
\text { (Subsidio a la } \\
\text { oferta) }\end{array}$ & Estado prestador \\
\hline
\end{tabular}

Fuente: Realización propia en base a Mainetti, J. L. (1998).

Cuadro 2 - Fortalezas y Debilidades del financiamiento a la oferta y a la demanda

\begin{tabular}{|c|c|c|}
\hline Estado & Fortalezas & Debilidades \\
\hline $\begin{array}{l}\text { Financiador de } \\
\text { la oferta }\end{array}$ & $\begin{array}{c}\text { No requiere cambios } \\
\text { tributarios } \\
\text { Genera menos resistencia } \\
\text { social }\end{array}$ & $\begin{array}{l}\text { El estado no sabe cuanto ni de que } \\
\text { forma gasta su presupuesto } \\
\text { Suele ser poco eficiente }\end{array}$ \\
\hline $\begin{array}{l}\text { Financiador de } \\
\text { la demanda }\end{array}$ & $\begin{array}{c}\text { Es más eficiente } \\
\text { Permite mejorar la macro y } \\
\text { la microasignación de los } \\
\text { recursos }\end{array}$ & $\begin{array}{l}\text { Requiere adecuaciones tributarias y } \\
\text { políticas } \\
\text { Si el sistema no está bien } \\
\text { implementado puede producir } \\
\text { exclusiones indeseadas }\end{array}$ \\
\hline
\end{tabular}

Fuente: Realización propia en base a Mainetti, J. L (1998).

El Cuadro 2 presenta las fortalezas y debilidades de cada opción. Las mismas deberían ser analizadas en el contexto de cada sistema. 
La particular situación de heterogeneidad y fragmentación de muchos sistemas es una fuente indudable de inequidad en la asignación de los recursos, aumentando la necesidad de transformación de la atención médica para que pueda dar respuesta satisfactoria a las demandas de su población.

En sistemas con una oferta amplia y altamente especializada, que por las características propias del mercado posee un grado mayor de información y bajo determinadas circunstancias alto poder de inducción no parece descabellado transmitir algo de poder a los demandantes.

De una u otra forma el Estado, por las características intrínsecas del mercado sanitario, donde las fallas son más que evidentes, debe jugar un papel activo para acercar la oferta a las necesidades de la demanda. Para ello debe utilizar toda la información disponible, incluso generando sistemas de información más amplios, que permitan realizar una correcta evaluación de las prioridades sanitarias.

\section{CONSIDERACIONES FINALES}

Dada la importancia del sector sanitario, tanto por volumen de gasto como por considerarse estratégico para el crecimiento y desarrollo de los países, es necesario un análisis exhaustivo de sus componentes, tarea que ha llevado a cabo una disciplina relativamente reciente: La economía de la salud.

Dentro de esta disciplina, el análisis de los componentes básicos de cualquier mercado, la oferta y demanda, hacen necesario su análisis con detenimiento, dado que el sector sanitario puede considerarse una "economía anormal".

La relación entre oferta y demanda en este sector dista bastante de lo que la teoría económica especifica para otros mercados. Las condiciones de salud de una población, que se reflejan en la demanda y utilización de servicios sanitarios, están especialmente ligadas a la estructura de la oferta de los mismos.

En cuanto a la oferta de servicios sanitarios no hay respuestas claras a los problemas teóricos planteados. La medición del producto, la variabilidad de la práctica médica, las diferencias de calidad y la eficiencia son temas aún no resueltos.

La posibilidad de los proveedores de influir sobre la demanda como consecuencia de la incertidumbre e información asimétrica, en un contexto donde se presentan dificultades para medir eficiencia y producto, unido a una gran variabilidad en los recursos y las prácticas utilizadas por los médicos para el diagnóstico y tratamiento de distintas enfermedades, hace esperable cierto grado de inducción de demanda. Aunque la existencia de seguros pone, bajo ciertas condiciones, límites a esta práctica, es necesario introducir ciertas restricciones a la oferta para limitar su influencia.

Más allá de la participación que el estado tenga en el sector, hay un rol que es ineludible: el de informador. Si gran parte de las fallas de mercado están dadas por problemas de información, crear flujos de información confiables, puede mejorar muchas situaciones. 
En caso de adoptar sistemas sanitarios predominantemente privados, sin correcta regulación, es bastante probable que las fuerzas de mercado permitan que la oferta pueda influir sobre la demanda y llegar a situaciones en las cuales los bienes y servicios prestados no se correspondan con las necesidades de la población. En este caso, el estado debe jugar un rol preponderante en el diseño de mecanismos de incentivos que contribuyan a mejorar estos resultados a partir de un conocimiento profundo de las condiciones básicas de oferta y demanda, de la estructura del mercado, de la conducta de los agentes que intervienen y de los resultados alcanzados. La definición de estos resultados esperados deberá ser producto de una priorización conciente de los objetivos deseados a la luz de las propias restricciones presupuestarias.

Cada sociedad debe estructurar su sistema de salud teniendo en cuenta sus condiciones básicas de oferta y demanda. No existen recetas universales que puedan pasar de un país a otro. Esto obliga a cada nación a construir su propio sistema sanitario en base a una evaluación profunda de sus recursos y necesidades. La dificultad que se plantea en muchos países es la ausencia de datos estadísticos que reflejen verazmente la situación en la que se encuentran para poder en base a ella construir modelos de atención, gestión y financiamiento acordes a sus necesidades.

Varios de los temas aquí tratados han sido y aún son eje central de las discusiones económicas dentro del sector. La Economía de la Salud no debe perder de vista que las consideraciones económicas de la práctica médica van unidas indefectiblemente a las decisiones médicas y mientras estas decisiones tengan un grado alto de variabilidad la intervención de la economía estará limitada a ciertos temas y encontrará dificultades para dar respuestas a algunos problemas importantes. Reconocer estas limitaciones es útil para avanzar.

\section{REFERENCIAS BIBLIOGRAFICAS}

Arredondo López, A, Recamann Mejía, A., (2002), “Oferta vs. Demanda: Algunos aspectos a considerar para el estudio del Mercado de Salud", Hitos de Ciencias económico administrativas, Nro. 20, pp.42-47.

Artells Herrero, J., (1999), "Necesidad vs.Demanda Sanitaria", Documento de Trabajo $\mathrm{N}^{\mathrm{o}} 11$, Fundación Salud, Innovación y Sociedad, Madrid.

Folland, S., Goodman, A. and Stano, M., (1997), The Economics of Health and Health Care, Prentice Hall, Upper Saddle River, New Jersey, Chapters 7 and 8.

Foster, B., (1989), "Optimal health investment strategies", Bulletin of Economics Research, Vol. 41, (1), pp.45-57.

Grossman, M., (1972), "The demand for health: A theorical and empirical investigation", NBER papers $\mathrm{N}^{\circ} 119$ New York.

Grossman, M, (1999), "The human capital model of the demand of health", Working paper 7078, NBER, Abril 1999.

Londoño, J. L.; Frenk, J., (1997), Pluralismo estructurado: hacia un modelo innovador para la reforma de los sistemas de salud en América Latina. In Frenk, J., ed., Economía y salud, necesidades, servicios y políticas. Fundación Mexicana para la Salud, México, DF, México. 
López, A. y Recaman Mejía, A. (2002), "Oferta vs. demanda: algunos aspectos a considerar para el estudio del mercado en salud", Hitos de ciencias económico administrativas, Nro.20, pp. 42-47.

Maceira, D., (1998), Mecanismos de pago a prestadores en el sistema de salud: incentivos, resultados e impacto organizacional en países en desarrollo. Agosto de 1998. Estudios Mayores de Investigación Aplicada 2, Documento de Trabajo 2. Bethesda, MD: Partnerships for Health Reform Project, Abt Associates Inc.

Mainetti, J. L., (1998), "El estado como financiador de la salud", Quirón Nro. 27, La Plata.

Mas-Colell, A. and Whinston, M, (1995), Microeconomic Theory, Oxford University Press, New York.

McGuire, T. and Pauly M., Physician responses to fee changes with multiple payers. Journal of Health Economics, $\mathrm{N}^{\mathrm{o}}$ 10, pp.385-410.

Phelps, C. and Newhouse J. (1971), "The Effects of Coinsurance on the Demand for Physician Services", The RAND Corporation, R-976-OEO.

Phelps, Ch., (1997), Health Economics, Addison-Wesley, New York.

Pohlmeier W. and Ulrich V., (1995), "An econometric model of a two part in a decisionmaking process in the demand for health care", The journal of human resources, Vol. 30, (2), pp.339-361.

Richardson J., (1981), "Supply and Demand for Medical Care: Or, Is the Health Care Market Perverse?, The Australian Economic Review, Vol. 34, (3), pp336-352.

Rochaix, Lise (1989), "Information Asymmetry and Search in the Market for Physician Services," Journal of Health Economics, No8, pp. 53-84.

Roemer, M. I., (1961), "Bed supply and hospital utilization", Journal of the American Hospitals Association, Vol. 35 , pp. 36-42.

Rossiter, L. and Wilensky, G., (1984), "Health Economist-Induced Demand for Theories of Physician-Induced Demand", Journal of Human Resources, Vol. 22 (4), pp. 624-627.

Shain, M. and Roemer, M., (1959), "Hospital Utilization under insurance", American Hospital Association, Monograph Series No 6 Chicago.

Tafani, R., (2000), Economía Sanitaria, Editorial de la Universidad Nacional de Río Cuarto, Argentina.

Temporelli, K. (2007), "Demanda secuencial de servicios sanitarios: la utilidad del médico como determinante" Ensayos de Economía de la Salud, Ed. Temas Argentina 2007.

Temporelli, K. y González, G.,(2008), "Elasticidad de los gastos sanitarios: un análisis a diferentes niveles de ingreso", Estudios de economía aplicada, Vol. 26, (1), 2008.

Wagstaff, A., (1986), "The demand for health: some new empirical evidence", Journal of Health economics, Vol. 5, (3), pp.195-233.

Weisbrod, B. A.,(1991), "The Health Care Quadrilemma: An Essay on Technological Change, Insurance, Quality of Care, and Cost Containment", Jounal of Economic Literature, №29. 